\title{
siRNA-mediated simultaneous downregulation of uPA and its receptor inhibits angiogenesis and invasiveness triggering apoptosis in breast cancer cells
}

\author{
RAMESH SUBRAMANIAN ${ }^{1}$, CHRISTOPHER S. GONDI ${ }^{1}$, \\ SAJANI S. LAKKA ${ }^{1}$, AMAN JUTLA ${ }^{1}$ and JASTI S. RAO ${ }^{1,2}$ \\ ${ }^{1}$ Division of Cancer Biology, Department of Biomedical and Therapeutic Sciences; \\ ${ }^{2}$ Department of Neurosurgery, University of Illinois College of Medicine at Peoria, IL, USA
}

Received August 31, 2005; Accepted November 18, 2005

\begin{abstract}
A wide variety of tumor cells exhibit overexpression of urokinase plasminogen activator (uPA) and its receptor ( $\mathrm{UPAR}$ ). In breast cancer, expression of uPA and UPAR is essential for tumor cell invasion and metastasis. It is also known that uPA binds to UPAR and activates the RAS extracellular signal regulated kinase (ERK) signaling pathway. In our study, small interfering RNA (siRNA) was introduced to downregulate the expression of UPA and UPAR in two breast cancer cell lines (MDA MB 231 and ZR 75 1). UPA and UPAR were downregulated individually using single constructs, and in combination using a bicistronic construct driven by a CMV promoter in a pcDNA-3 mammalian expression vector. Reverse transcription PCR (RT-PCR) and Western blot analyses indicated downregulation at both the mRNA and protein levels. In vitro angiogenesis studies using conditioned medium in HMEC-1 cells indicated a decrease in the angiogenic potential of conditioned media from treated cells when compared to the controls. This decrease in angiogenic potential was remarkably higher with the bicistronic construct. Similarly, the invasive potential of these cells decreased dramatically when treated with the bicistronic construct, thereby suggesting a synergistic effect from the downregulation of both UPA and uPAR. Furthermore, when uPA and UPAR were downregulated simultaneously, the apoptotic cascade was triggered as indicated by the upregulation of both initiator and effector caspases as well as other pro-apoptotic molecules. A
\end{abstract}

Correspondence to: Dr J.S. Rao, University of Illinois College of Medicine, 1 Illini Drive, Peoria, IL 61656, USA

E-mail: jsrao@uic.edu

Abbreviations: RNAi, RNA interference; siRNA, short-interfering RNA; uPA, urokinase-type plasminogen activator; uPAR, uPA receptor; ECM, extracellular matrix; SV, scrambled vector; GAPDH, glyceral dehyde-3-phospho dehydrogenase; PBS, phosphate-buffered saline; CMV , cytomegalovirus

Key words: breast cancer cells, invasion, angiogenesis, uPA, uPAR mitochondrial permeability assay and FACS analysis revealed an increase in apoptotic cells in the UPA/uPAR treatment as compared to the other treatments. This overexpression of pro-apoptotic caspases in relation to the RNAi-induced downregulation of UPA and UPAR clearly suggests the involvement of the UPA-uPAR system in cell survival and proliferation in addition to their role in tumor progression.

\section{Introduction}

In multicellular organisms, invasion plays a pivotal role in several diverse physiological and pathological processes including tissue remodeling associated with embryonic development (1), inflammation (2,3), angiogenesis (4), wound healing (5), and tumor metastasis (6-9). Among the large number of proteases involved in invasion, urokinase plasminogen activator (UPA) and urokinase-specific cell surface receptor (UPAR) are of particular importance (10-12). $\mathrm{UPA}$ is a serine protease and when bound to its receptor, UPAR, initiates the activation of metalloproteases as well as the conversion of plasminogen to plasmin $(13,14)$. In addition, it has been widely reported that the ability of uPAR to localize the proteolytic activity of uPA on the cell surface is extremely important for the invasive ability of tumor cells (15). In addition to its proteolytic function as a zymogen, the uPA/ uPAR complex functions as a vitronectin receptor and has been shown to participate in normal and tumor cell motility processes including monocyte migration and tumor cell migration and invasion (16-18). The major change associated with cellular transformation is the regulation of mitogenic pathways, which become constitutively activated (19). Oncogenic cells show a dramatic enhancement of protease production (e.g. uPA), which may promote increased pericellular proteolysis leading to the release of angiogenic and growth factors.

As such, the uPA/uPAR system presents a viable therapeutic target for anti-cancer studies. We have developed siRNA constructs for the inhibition of these proteins. RNA interference is the process of sequence-specific posttranscriptional gene silencing mediated by double-stranded RNA molecules (21-23 bp in length) homologous to the sequence of the silenced gene. Elbashir et al (20) showed that 
synthetic 21-23 nucleotide siRNA could induce efficient RNA interference in mammalian cells. In order to circumvent the high cost of synthetic siRNA and establish gene knockdown cell lines by siRNA, several plasmid vector systems have been designed to produce siRNA inside cells. These siRNA sequences are driven by RNA polymerase III-dependent promoters such as U6 and H1-RNA. In addition, siRNA has been shown to be potent since only a few molecules of dsRNA per cell are necessary to trigger gene silencing throughout the treated animal (21). Earlier studies in our laboratory have successfully demonstrated the efficiency of CMV promoter-driven siRNA sequences in the downregulation of ECM-degrading proteases (22). In the current study, we selected two breast cancer cell lines (MDA MB 231, an aggressively invasive cell line with high levels of uPA and UPAR, and ZR 75 1, a moderately aggressive cell line with moderate levels of uPA and uPAR expression).

Earlier studies with MDA MB 231 cells have shown the use of UPAR antagonists and UPA inhibitors to downregulate the invasive ability of breast cancer cells (23). We present evidence of RNAi-induced downregulation of uPA and uPAR at both the mRNA and protein level by reverse transcription PCR and Western blot analysis respectively. Furthermore, we show that when UPA and UPAR are downregulated, the invasiveness and angiogenic potential of cancers are inhibited. The uPA/uPAR system not only plays a role in proteolytic cleavage of the ECM but also plays a significant role in the signaling involved in cell survival and proliferation. Here, we demonstrate that inhibition of this signaling triggers the apoptotic cascade.

\section{Materials and methods}

Cell lines and culture conditions. The two breast cancer cell lines (MDA MB 231 and ZR 75 1) were obtained from ATCC (Manassas, VA). As suggested by ATCC, MDA MB 231 cells were grown in L-15 medium supplemented with glutamine, $10 \% \mathrm{FBS}, 100 \mu \mathrm{g} / \mathrm{ml}$ streptomycin, and $100 \mathrm{units} / \mathrm{ml}$ penicillin (Invitrogen, Carlsbad, CA) at $37^{\circ} \mathrm{C}$. ZR 751 cells were maintained in RPMI-1640 medium supplemented with $10 \%$ FBS, $10 \mathrm{mM}$ HEPES, $1 \mathrm{mM}$ sodium pyruvate, $4.5 \mathrm{~g} / 1$ glucose, $1.5 \mathrm{~g} / 1$ sodium bicarbonate, $100 \mu \mathrm{g} / \mathrm{ml}$ streptomycin, and 100 units/ml penicillin. Human microvascular endothelial cells (HMEC) were cultured with advanced DMEM supplemented with $2 \% \mathrm{FBS}, 100 \mu \mathrm{g} / \mathrm{ml}$ streptomycin, 100 units $/ \mathrm{ml}$ penicillin, $1 \mu \mathrm{g} / \mathrm{ml}$ hydrocortisone and $10 \mathrm{mM}$ EGF.

siRNA vectors. Small interfering RNA sequences targeting UPAR and UPA were introduced into a mammalian expression vector pcDNA-3 downstream of the cytomegalovirus promoter. An uPAR sequence $(+348$ to +369$)$ was used as the target sequence, and for convenience, a self-complimentary oligo was used. An uPAR sequence that was 23 bases in length with a 9base loop region with BamHI sites incorporated at the ends (gatcctacagcagtggagagcgattatatataataatcgctctccactgctgtag) was used. The self-annealed oligo was ligated into the BamHI site of pcDNA-3 vector. Similarly, a uPA sequence $(+78$ to +98$)$ was used as the target sequence. The 21-base uPA sequence with a 9-base loop sequence incorporated between the repeats and HindIII site on either end (agctgagagccetgctggcgcgcca tatataatggcgcgccagcagggctctca) was used. The bicistronic construct carried both the UPAR and uPA sequences and the BamHI and HindIII sites respectively. BGH poly-A terminator served as a stop signal for RNA synthesis for all three constructs. The vectors carrying UPAR and UPA were named $\mathrm{pU}$ and pUPA respectively and the bicistronic construct was named pU2. The plasmid vectors were cloned and maintained in JM109 cells. A scrambled sequence of nucleotides was also introduced into the expression cassette and used as a mock vector (pSV). Plasmid DNA was isolated using the Qiagen plasmid purification system and analyzed for quality and quantity (Qiagen, Valencia, CA).

Transfection studies. Cells $\left(1 \times 10^{6}\right)$ were plated in $100 \mathrm{~mm}$ Petri dishes for each transfection experiment. The cells were transfected in serum-free L-15 media using $10 \mu \mathrm{g}$ of lipofectamine reagent (Invitrogen, Grand Island, NY) as per the manufacturer's instructions. The pU, pUPA, pU2 and pSV constructs were used for transfection. No plasmid was introduced in the control plates. After allowing $12 \mathrm{~h}$ for transfection, the serum-free media was replaced with serumcontaining media and left in the incubator at $37^{\circ} \mathrm{C}$ for $24 \mathrm{~h}$. The media was then replaced with serum-free media and conditioned media was collected after $12 \mathrm{~h}$. Cells were harvested for isolation of total RNA and/or total cell lysate. Conditioned media was used for fibrin zymography and in vitro angiogenic assay.

$R T-P C R$. Total RNA was isolated from control and transfected cells using the PARIS ${ }^{\mathrm{TM}}$ protein and RNA isolation system (Ambion, Austin, TX). Total RNA was treated with DNase I (Invitrogen). First strand cDNA was prepared using SuperScript III reverse transcriptase (Invitrogen) using oligo dT. First strand cDNA (100 ng) was used for PCR amplification. We used the following primers for uPAR: uPAR-S- CATGC AGTGTAAGACCCAACGGGGA and uPAR-AS- AATAG GTGACAGCCCGGCCAGAGT. The following primers were used for uPA: uPA-S- TGCGTCCTGGTCGTGAGCGA and uPA-AS- CAAGCGTGTCAGCGCTGTAG. GAPDH was used as an internal control. PCR analysis was carried out using puReTaq ${ }^{\mathrm{TM}}$ Ready-To-Go PCR beads (Amersham Biosciences, UK).

Western blot analysis. MDA MB 231 and ZR 751 cells were transfected with pSV, pU, pUPA and pU2 as described above. At the end of the designated incubation period, cells were harvested, washed twice with cold PBS, and lysed in a buffer (150 mM NaCl, 50 mM Tris-HCl, 2 mM EDTA, 1\% NP-40, $\mathrm{pH}$ 7.4) containing protease inhibitors. Equal amounts of protein $(20 \mu \mathrm{g} /$ lane $)$ from cell lysate were electrophoresed under non-reducing conditions on $10 \%$ acrylamide gels. After SDS-PAGE, proteins were transferred to a polyvinylidene difluoride membrane (BioRad). The membrane was incubated for $2 \mathrm{~h}$ in PBS [0.1\% Tween-20 (T-PBS), 5\% nonfat skim milk] to block non-specific binding. Subsequently, the membrane was incubated for $2 \mathrm{~h}$ with antibody against UPAR (R\&D Systems); ERK, pERK, p38, p-p38 (all, Santa Cruz Biotech Inc.); caspases 9, 8, 6 and 3 (Cell Signal); and APAF-1 (MBL International) respectively in T-PBS $+5 \%$ non-fat milk. After washing in T-PBS, proteins were visualized using the 


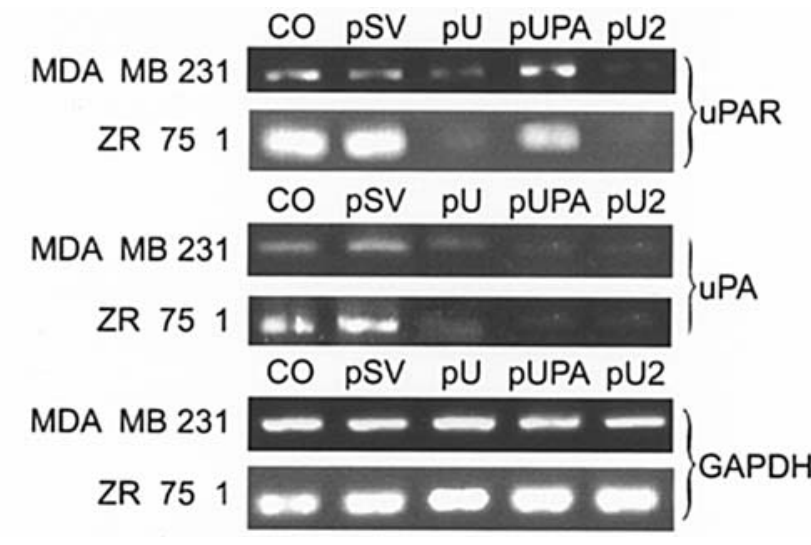

Figure 1. Reverse transcription PCR analysis of RNAi transfected breast cancer cells. Cells $\left(1 \times 10^{6}\right)$ were plated on $100 \mathrm{~mm}$ petri plate for each transfection experiment. Cells were transfected with $\mathrm{CO}$ (control with no plasmid). Scrambled vector (pSV), uPAR (pU), pUPA and pU2. After $48 \mathrm{~h}$, total RNA was isolated from the cells to prepare first strand cDNA followed by PCR analysis using the primers for uPA and UPAR. GAPDH was used as an internal control.

ECLTM detection kit with appropriate HRP-conjugated secondary antibody (Amersham Pharmacia Biotech, UK) as per the manufacturer's instructions. The membranes were stripped and probed with monoclonal antibodies for GAPDH for loading control as per standard protocols.

In vitro angiogenesis. Conditioned media collected from transfected MDA MB 231 and ZR 751 cells was tested for induction of angiogenesis in human microvascular endothelial cells (HMEC) plated onto 8-well chamber slides (4x104/well). Once the cells had attached to the surface of the slide, the media was removed and replaced with conditioned media collected from RNAi-transfected cells. After $72 \mathrm{~h}$ in conditioned media, cells were washed gently with PBS and stained with hematoxylin and eosin (H\&E). Angiogenic network formation was visualized under a microscope and Image Pro software was used for quantification of angiogenesis. The degree of angiogenesis was measured by the following method: number of branch points and the total number of branches per point were counted at random (per 10 fields), with the product indicating the degree of angiogenesis as compared to the controls.

Boyden chamber invasion assay. The in vitro invasiveness of MDA MB 231 and ZR 751 cells in the presence of vectors expressing siRNA for UPAR and UPA individually and in combination was assessed using a modified Boyden chamber assay. Breast cancer cells were transfected with $\mathrm{pSV}, \mathrm{pU}$, pUPA and pU2 vectors as described above. After allowing $24 \mathrm{~h}$ for transfection, $5 \times 10^{5}$ cells were suspended in $600 \mu \mathrm{l}$ of serum-free medium and placed in the upper compartment of the transwell chambers [12 $\mu \mathrm{m}$ pore size, $12 \mathrm{~mm}$ diameter (Corning Costar Fischer Scientific, Pittsburg, PA)] coated with matrigel $\left(100 \mu \mathrm{g} / \mathrm{cm}^{2}\right)$. The lower compartment of the chambers was filled with $200 \mu$ l of serum-containing medium and the cells were allowed to migrate for $24 \mathrm{~h}$. After incubation, the cells were fixed and stained with Hema-3 and photographed. Quantification of the invasion assay was performed as described previously (24-26).
MITO-PT 100 and FACS analysis. Induction of apoptosis in response to RNAi-induced downregulation of UPAR and uPA individually and in combination was tested using the MITO-PT 100 assay (Serotec). MDA MB 231 and ZR 751 cells were transfected with pSV, pU, pUPA and pU2 as described above. After $72 \mathrm{~h}$, the cells were trypsinized and pelleted. The cells were then resuspended in MitoPT dye diluted in serum-free media as per manufacturer's instructions. After an incubation period, $100 \mu \mathrm{l}$ of the cell suspension was visualized under a fluorescent microscope. MitoPT kit allows distinction between non-apoptotic (red fluorescence) and apoptotic (green fluorescence) cells. The remaining cells were used for FACS analysis. FACS analysis was performed according to the manufacturer's instructions and 10,000 cells were recorded for each transfection. When cells stained with MitoPT are run through a flow cytometer, the instrument measures apoptosis by monitoring the amount of red fluorescence in each region. As the mitochondrial membrane collapses, a reduction in the amount of red fluorescence is observed as MitoPT dye is converted from a dimer form to a monomer form. These experiments were performed in triplicate for each cell line.

\section{Results}

$R T-P C R$. In order to study the effects of downregulation of $\mathrm{UPA}$ and UPAR, individually and in combination, on tumor cell-induced angiogenesis, cancer cell invasion and survival, we transfected breast cancer cell lines MDA MB 231 and ZR 751 with pcDNA mammalian expression vectors harboring small interfering RNA sequences driven by a CMV promoter. mRNA levels were determined using semi-quantitative RT-PCR. Briefly, $48 \mathrm{~h}$ after transfection, total RNA was isolated from the cells and first strand cDNA was prepared using SuperScript III reverse transcriptase and oligo dT. PCR analysis was carried out using the appropriate primers for UPAR and uPA. Clear amplification was observed in the control and scrambled vector at approximately $280 \mathrm{bp}$ with the primers for uPAR, and at 780 bp with the primers for uPA. While no change was observed in the levels of amplified product in cells treated with the scrambled vector as compared to the control, significant decreases in the levels of uPAR was observed in treatments with pU and pU2 (Fig. 1). Similarly, uPA levels were significantly decreased in pUPA and pU2 when the primers for uPA were used. The levels of GAPDH remain unchanged in all the treatments, indicating uniform levels of first strand cDNA taken in all the cases.

UPAR and UPA protein expression. The levels of UPAR protein in the total cell lysate and uPA in the conditioned media was assessed by Western blot and fibrin zymography respectively. Western blot analyses using the anti-uPAR antibody revealed significant decreases in uPAR expression after transfection with pU in MDA MB 231 and ZR 751 cells (Fig. 2A). However, no change in UPAR expression was observed after transfection with pUPA levels when compared to the control (Fig. 2A). Quantitative analysis of UPAR protein by densitometry revealed a decrease in protein expression with pU by 40 and $60 \%$ and in pU2 by 75 and $80 \%$, respectively for MDA MB 231 and ZR 751 compared to controls and pSV and pUPA transfected cells (Fig. 2B). 

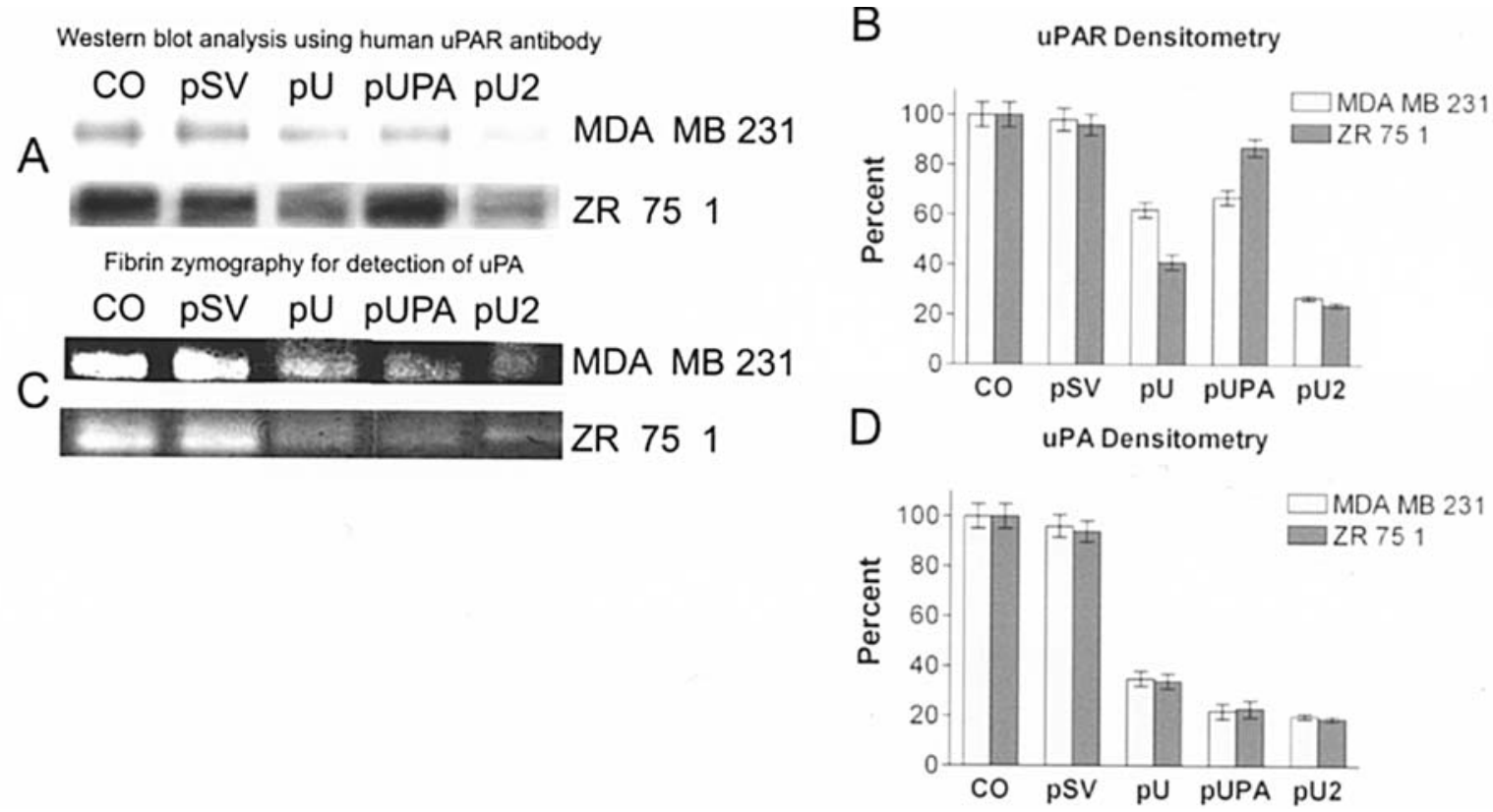

Figure 2. Western blot analysis and fibrin zymography. Transfection was carried out as described earlier. Cells $\left(1 \mathrm{x} 10^{6}\right)$ were plated on $100 \mathrm{~mm}$ petri plate for each transfection experiment. Cells were transfected with CO (control with no plasmid). Scrambled vector (pSV), uPAR (pU), pUPA and pU2. After 36 h, serum-containing media from the plate was replaced with serum-free media. After $48 \mathrm{~h}$, cells were harvested for protein extraction and conditioned media was collected $20 \mu \mathrm{g}$ of total protein from each of the samples was loaded on SDS-PAGE and blotted on nitrocellulose membranes. The membrane was probed with anti-uPAR antibody (A). Conditioned media $(0.2 \mu \mathrm{g})$ was loaded for fibrin zymography (C). Quantification of uPAR protein (B) and uPA enzymatic activity (D) was obtained by scanning the auto-radiograms with a densitometer. Data are shown mean values \pm SD of three different experiments in each group.

Fibrin zymography using conditioned medium from treated cells revealed a decrease in the levels of uPA activity in all the treatments as compared to the control and SV-treated group (Fig. 2C). Treatment with pUPA and pU2 showed uPA activity was decreased, ranging from 77 to $81 \%$ in MDA MB 231 and ZR 751 cells, respectively. pU treatment also showed reduced uPA activity of 65 and 66\% (Fig. 2D) in MDA MB 231 and ZR 751 cells, respectively. No significant change was observed in the case of scrambled vector when compared to the control.

Inhibition of angiogenesis in HMEC-1 cells. Tumor cells induce angiogenesis in order to maintain the flow of nutrients for the growing number of cells. Since uPAR has been implicated in the regulation of angiogenesis, we assessed the effects of pU, pUPA and pU2 on tumor cell-induced angiogenesis. HMEC, grown in conditioned media from MDA MB 231 cells, and ZR 751 cells transfected with pSV, pU, pUPA, pU2 or untransfected control were stained with H\&E. The results clearly demonstrated that endothelial cells form capillary-like structures in the presence of control and pSVtransfected cells within $72 \mathrm{~h}$, in the case of conditioned media from MDA MB 231 cells (Fig. 3A), and within $96 \mathrm{~h}$ in the case of ZR 751 cells (data not shown). However, pU2 significantly inhibited tumor cell-induced angiogenesis. The quantification of the branch points and number of branches was almost undetectable in conditioned media from pU2-transfected cells, which decreased $94 \%$ with conditioned media from MDA MB 231 cells and $92 \%$ with conditioned media from ZR 751 cells as compared to the control (Fig. 3B). Conditioned media from pU- and pUPA-treated cells also demonstrated decreases in tumor cell-induced angiogenesis ranging from
$60 \%$ for pU in ZR 751 to $70 \%$ in pUPA-transfected MDA MB 231 (Fig. 3B).

Inhibition of breast cancer cell invasion by $p U, p U P A$ and $p U 2$. To study the effect of siRNA-mediated downregulation of UPA and UPAR on their invasive potential, breast cancer cell lines MDA MB 231 and ZR 751 were transfected with pU, pUPA, pU2, or pSV and transferred to matrigel-coated transwell chambers. Extensive invasion was observed within $48 \mathrm{~h}$ in the untransfected controls and cells transfected with pSV (Fig. 4A). Fig. 4A illustrates that the staining of pU, pUPA and pU2 transfected MDA MB 231 and ZR 251 cells was significantly less than that of the controls (Fig. 4A). In MDA MB 231 cells, pU2 treatment resulted in the least number of invading cells with $12 \%$ of the control while pUPA and pU treatment resulted in $19 \%$ of the control. In ZR 751 cells, inhibition of invasion was observed to a lesser extent with pU and pUPA treatments showing a $<50 \%$ invasion as compared to the control. Once again, maximum inhibition was observed with pU2 treatment with only $21 \%$ invasion when compared to the control (Fig. 4B). Invasion of cells transfected with pSV in both MDA MB 231 and ZR 751 cells did not differ significantly from the controls.

It has been reported previously that antibody-mediated inhibition of uPA induces the expression of caspase 3 . To study the induction of apoptotic cell death due to downregulation of UPA and UPAR, FACS analysis was performed using the MitoPT $^{\mathrm{TM}}$ mitochondrial permeability transition detection kit. After $72 \mathrm{~h}$ of transfection, the cells were run through a flow cytometer. In the cells transfected with $\mathrm{pSV}, \mathrm{pU}$ and $\mathrm{pUPA}$, the majority of the cells were seen to fall in the red fluorescent region, indicating the presence of intact mitochondria. 

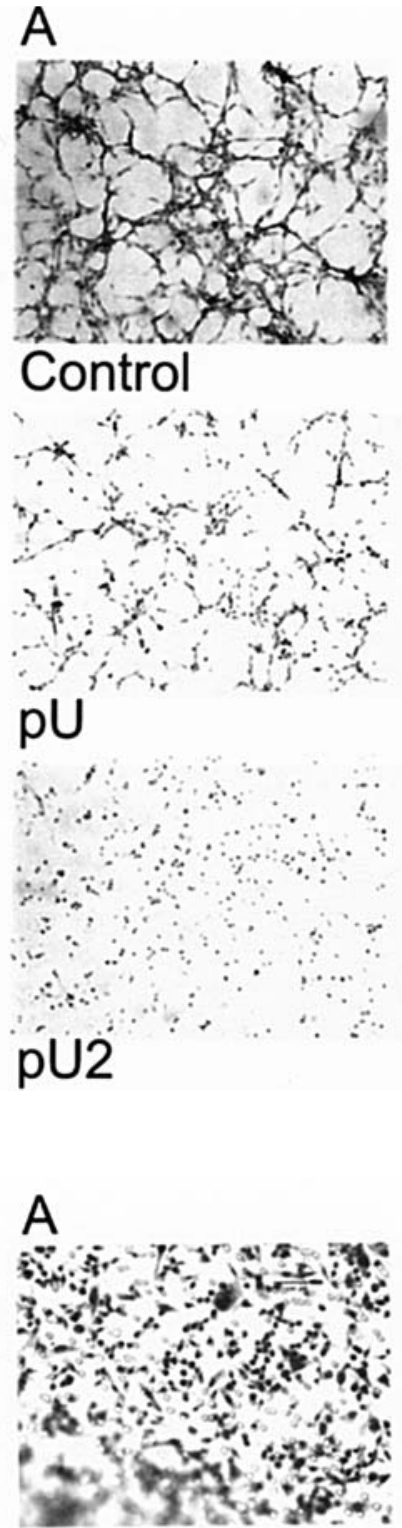

Control

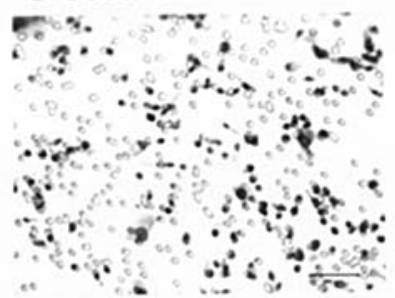

$\mathrm{pU}$

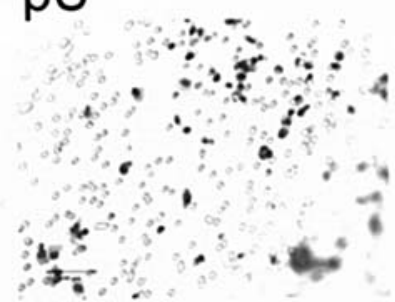

pU2
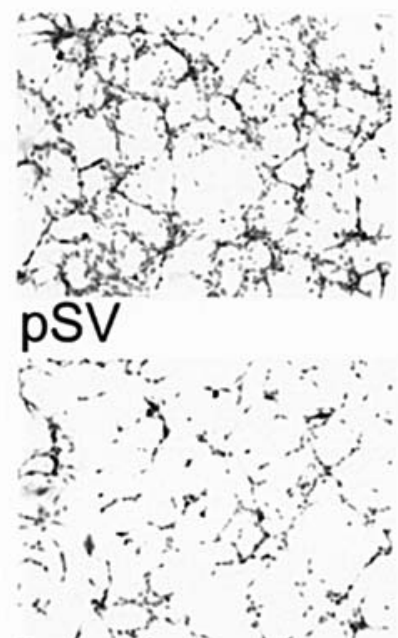

pUPA
B

\section{Tumor Cell Induced Angiogenesis}

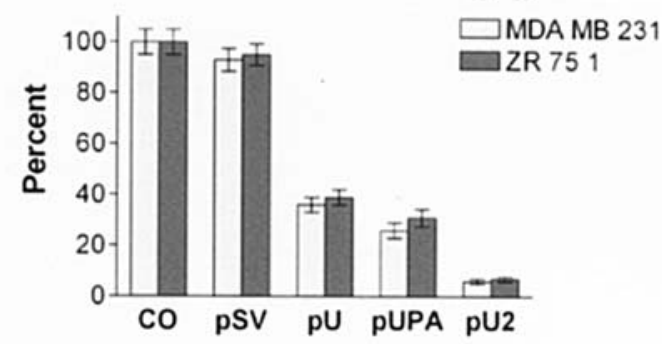

Figure 3. In vitro angiogenesis. Network formation by human microvascular endothelial cells in conditioned media from MDA MB 231/ZR 751 cells. Breast cancer cells were transfected with control $(\mathrm{CO})$, scrambled vector (pSV) or RNAi constructs for uPAR (pU), uPA (pUPA), uPAR-uPA (pU2) and conditioned media was collected after $48 \mathrm{~h}$ as described earlier. HMEC-1 cells grown in conditioned media were stained with $\mathrm{H} \& \mathrm{E}$ after $72 \mathrm{~h}$ and examined under a confocal scanning laser microscope (A). Quantification of angiogenesis in co-cultures transfected with $\mathrm{pSV}, \mathrm{pU}, \mathrm{pUPA}$ and $\mathrm{pU} 2$ as described in Materials and methods. Values are mean \pm SD from three different experiments.
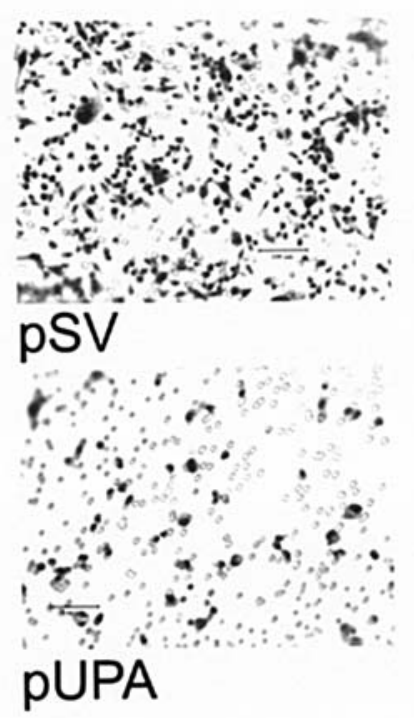

Figure 4. Matrigel invasion assay. Breast cancer cells transfected with control (CO), scrambled vector (pSV), or RNAi constructs for uPAR (pU), uPA (pUPA), uPAR-uPA (pU2) were transferred to $12 \mu \mathrm{m}$ transwell chambers coated with matrigel. After $48 \mathrm{~h}$, the cells were scraped from the chamber and stained to check for invasion of cells to the lower surface (A). The invasion was quantified as described in Materials and methods (B). Values are mean $\pm \mathrm{SD}$ of four experiments.

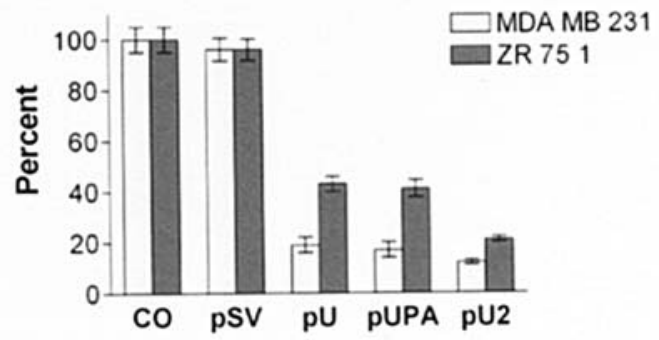

However, in the case of cells transfected with pU2, a significant drop in the number cells showing red fluorescence was observed, and also, a large number of events in the smaller size region was observed indicating the presence of
B

pU2-transfected cells could be clearly seen with more number of cells showing green fluorescence (Fig. 5B). As noted earlier, a large number of smaller particulate matter was observed in pU2-transfected cells as compared to the 

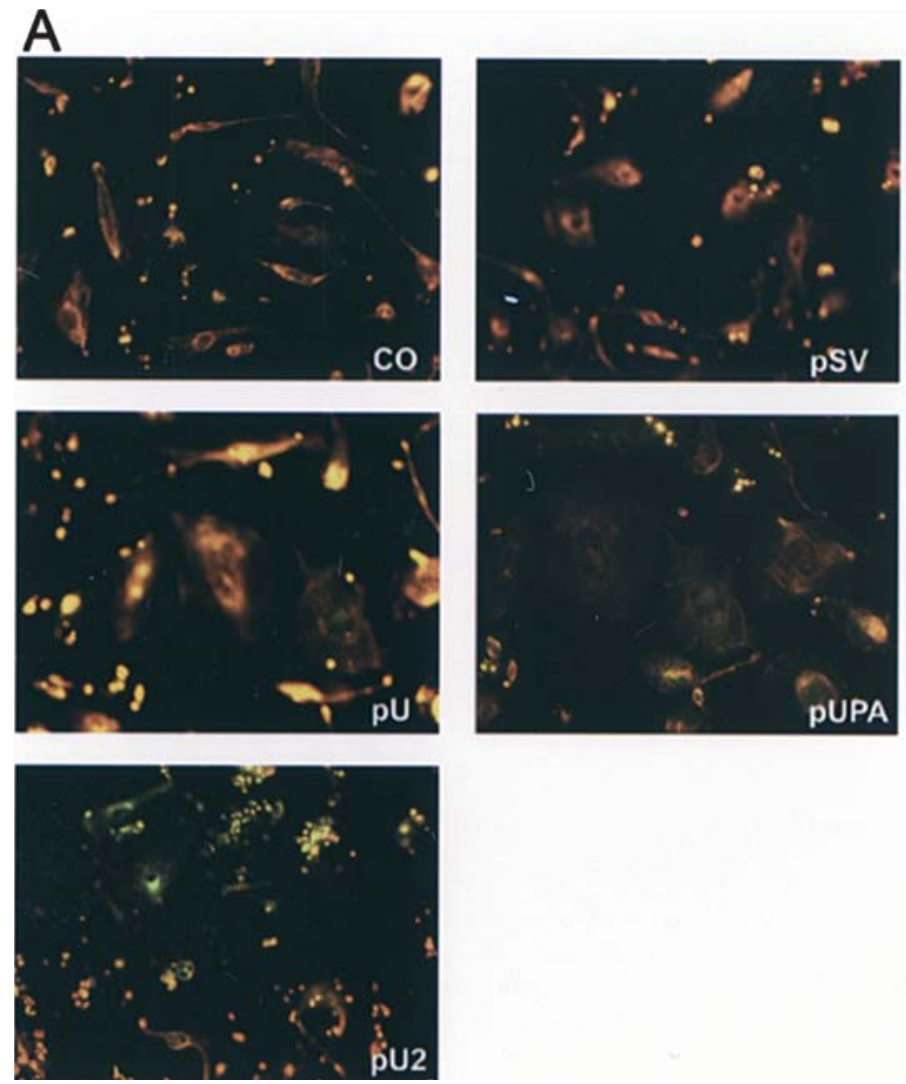

control indicating the presence of extensive cellular degradation.

To study the effect of simultaneous downregulation of uPAR and uPA on apoptotic cascade signaling, Western blot analysis was performed. Specifically, we tested for the expression of initiator and effector caspases as well as other genes involved in the apoptotic cascade. pU2 induced the expression of several genes involved in apoptosis, namely initiator caspases 9 and 8, and effector caspases 3 and 6 (Fig. 6). All the caspases mentioned above showed a 1.5-2-fold increase in pU2-transfected cells as compared to the control. We also observed a significant increase in the expression level of APAF-1, which is involved in apoptosome formation, in both cell lines after pU2 transfection. However, pUPA- and pU-transfected cells did not exhibit any changes in caspase expression level with the exception of caspase 9, which was seen to increase in pUPA-transfected MDA MB 231 cells. In addition to the increase in the expression levels of the genes involved in programmed cell death, changes were observed in the expression of proteins involved in cell survival. Significant decreases in the levels of phospho-ERK and phospho-p38 were observed in cells treated with pUPA and pU2, while the inactive non-phosphorylated forms remained unchanged when compared to control and pSV- and pU-transfected cells.

\section{Discussion}

Tumor cell invasion is associated with the destruction of the basement membrane and extracellular matrix. This process is associated with proteases, such as uPA and MMPs, secreted by malignant cells and stromal cells (6-9). Overexpression of $\mathrm{UPA}$ and $\mathrm{UPAR}$ has been well documented in a wide variety

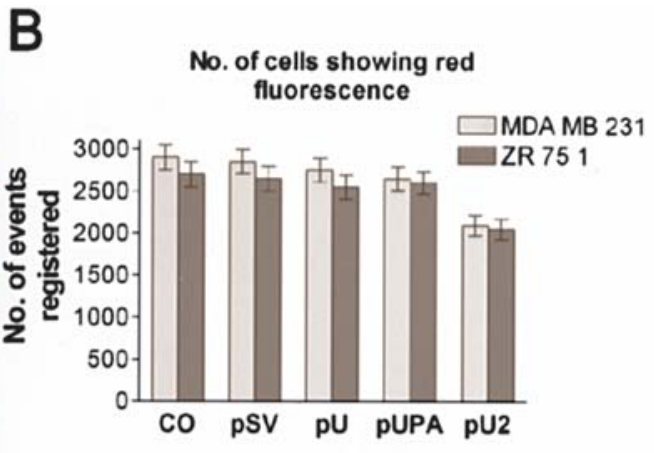

Figure 5. MITO-PT 100 and FACS analysis. MDA MB 231 cells were transfected with control $(\mathrm{CO})$, scrambled vector $(\mathrm{pSV})$, or RNAi constructs for $\mathrm{uPAR}(\mathrm{pU})$, uPA (pUPA), uPAR-uPA (pU2). After $48 \mathrm{~h}$, the cells were resuspended in MitoPT dye diluted in serum-free media. After incubating at $37^{\circ} \mathrm{C}$ for $15 \mathrm{~min}, 100 \mu \mathrm{l}$ of the suspension was visualized under a fluorescent microscope (A). MitoPT kit allows the distinction between non-apoptotic red fluorescent cells and apoptotic green fluorescent cells. FACS analysis was carried out according to the manufacturer's instructions and 10,000 cells were recorded for each transfection. When cells stained with MitoPT are run through a flow cytometer, the instrument will measure apoptosis by monitoring the amount of red fluorescence in each region (B).

of tumor cells. The urokinase plasminogen activator system has pleiotropic roles in tumor growth, angiogenesis and metastasis (27). In breast cancer, expression of uPA/uPAR is essential for tumor cell invasion and metastasis (28). Downregulation of matrix-degrading proteases and their receptors has potential therapeutic application in breast cancer. Antisense DNA and oligonucleotides are a convenient approach for the selective downregulation of protein expression (22). In a recent study, siRNA was quantitatively more efficient than antisense oligo-nucleotides at suppressing co-transfected GFP expression both in vitro and in vivo (29). Limitations to the use of RNAi in mammalian cells derive from unwarranted secondary responses to long dsRNA, such as induction of interferon synthesis (30). The recent development of 21-nucleotide siRNA duplexes has circumvented these problems and allowed successful RNAi in cultures of several types of mammalian cells (20). An important application of RNAi in mammals is the simultaneous inhibition of multiple genes in somatic cells. Previous observations demonstrated that there is no significant competition between co-transfected hairpin siRNA vectors.

In the present study, we have introduced siRNA sequences driven by a CMV promoter into breast cancer cell lines MDA MB 231 and ZR 751 to downregulate the expression of uPA and its receptor, individually and in combination, to assess the effects on cancer cell invasion, angiogenesis, survival and proliferation. Our results demonstrate that siRNA sequences can specifically reduce mRNA levels of uPAR and uPA without affecting the levels of housekeeping genes. Semiquantitative RT-PCR analysis also revealed that when UPAR was downregulated, a decrease in the mRNA levels of uPA was also noticed. However, the reverse was not observed, suggesting transcriptional regulation of uPA expression by uPAR. Fibrin zymography also revealed a decrease in levels of UPA when compared to the control. 

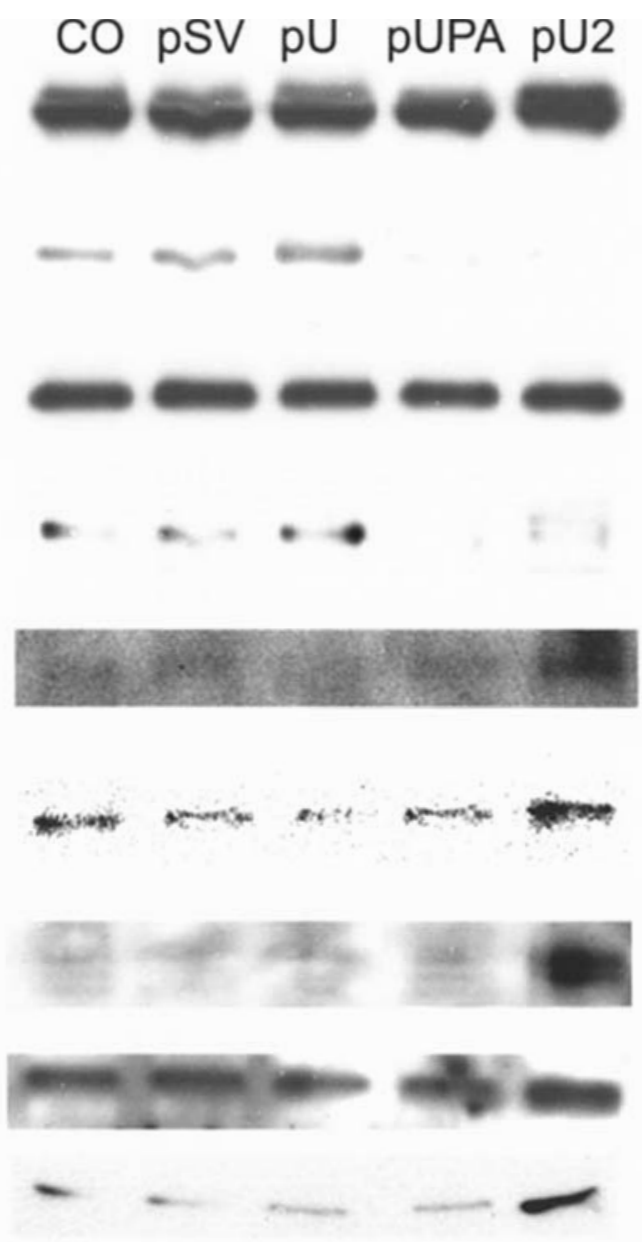

MDA MB 231
ERK

pERK

P38

pP38
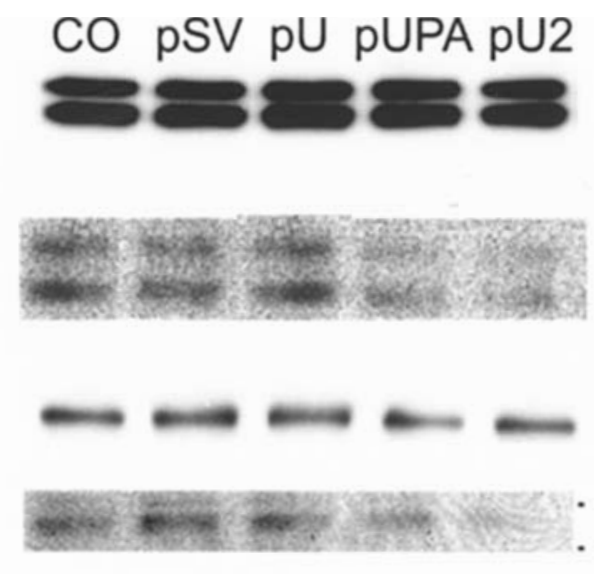

Caspase 3

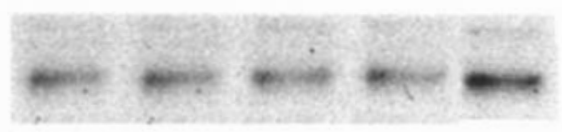

Caspase 6

Caspase 8

Caspase 9

APAF-1
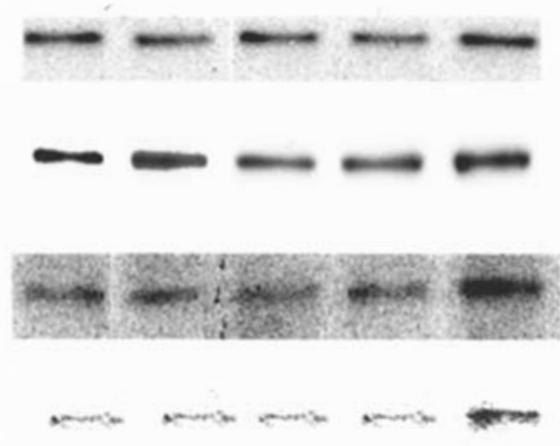

ZR 751

Figure 6. Simultaneous inhibition of uPAR-uPA induces apoptotic cascade. Breast cancer cells were transfected with control (CO), scrambled vector (pSV), or RNAi constructs for uPAR (pU), uPA (pUPA), uPAR-uPA (pU2). After $48 \mathrm{~h}$, cell lysates were prepared as per standard protocol and $15 \mu \mathrm{g}$ of total protein was loaded on SDS-PAGE and blotted on nitrocellulose membrane. The membrane was probed with antibodies specific for ERK, pERK, p38, pP38, Caspases 3, 6, 8 and 9 and APAF-1.

When conditioned media from transfected cells was assessed for angiogenic potential in HMEC, a significant decrease was seen in the number of branch points with conditioned media from pU- and pUPA-treated cells. The most remarkable decrease was observed in cells treated with pU2 where angiogenesis was reduced to $<10 \%$ of the control in both cell lines. This decrease clearly indicates a synergistic rather than cumulative effect of the co-suppression of uPAR and uPA.

Li et al (31) have reported the inhibition of neovascularization in MDA MB 231 xenografts introduced into athymic mice, when uPA/uPAR antagonists were used. In another study, Min et al (32) have reported the inhibition of angiogenesis in an in vitro model when high-affinity murine urokinase receptor antagonists were used. Li et al (31) have suggested that the inhibition of angiogenesis observed in mice treated with UPAR antagonist might have occurred because of the hampering of bioavailability of angiogenic factors. The pattern of angiogenesis was similar with conditioned media from both breast cancer cell lines tested. The dramatic decrease in in vitro angiogenesis observed in our study when both UPAR and UPA were downregulated simultaneously suggests a signaling-induced downregulation in the release of angiogenic factors.

Both in vitro and in vivo studies indicate that $\mathrm{UPA}$ and UPAR levels are closely associated with the degree of tumor cell invasion and that UPA and UPAR play key roles in tumor progression and metastasis. Expression of uPA and UPAR can be upregulated by mitogens, growth factors, oncogenes, and binding of integrin to extracellular matrix proteins. Generally, factors that upregulate uPA also upregulate UPAR, suggesting that UPA and UPAR may be co-regulated in cancer cells. We have observed that downregulation of UPAR exhibits a concomitant downregulation of uPA. We tested the effect of RNAi-induced downregulation on the invasive abilities of the MDA MB 231 and ZR 751 cancer cell lines. A similar pattern was observed in the invasive abilities of transfected cells when compared to control and scrambled vector, with only pU2-treated cells showing 12 and $21 \%$ invasion when compared to control in MDA MB 231 and ZR 751 cells, respectively.

uPA has been reported to induce chemotaxis and chemokinesis in a range of different cell types (33-39). Binding to uPAR seems to be required for all chemotaxic effects of uPA. 
The phenomena can be divided into two groups depending on whether uPA catalytic activity is also required. The binding of UPA to UPAR in some cell types causes the transmission of signaling over the cell membrane leading to increased motility (40). This ligand engagement of UPAR has been reported to cause the transient activation of a variety of signaling proteins including src-family of members $(37,41,42)$, ERK-1 and ERK-2 (36,43), FAK $(44,45)$, fos, jun and myc (46), and rac (47). In fact, inhibitors of these pathways block the effects of uPA (40). We observed that the levels of phospho-ERK and phospho-p38 in pU2-transfected cells in both breast cancer cell lines showed a significant decrease when compared to the control. The decrease in the expression levels of these prosurvival kinases can also be correlated with a significant increase in the levels of the pro-apoptotic caspases. A number of studies have demonstrated that activated ERK inhibits apoptosis (48-50). Western blot analysis revealed significant increases in levels of initiator and effector caspases in both breast cancer cell lines. While caspase 9 levels were seen to increase in pUPA, all the caspases tested were seen to increase significantly with pU2. We also observed an increase in APAF-1, the cytosolic protein which is the human homolog of the $C$. elegans CED-4. APAF-1 functions downstream to Bcl-2 (or its close family member $\mathrm{BcLX}_{\mathrm{L}}$ ), which regulates the release of cytochrome $\mathrm{c}$ from mitochondria (51). Cecconi et al (52) have suggested an in vivo apoptotic pathway whereby apoptotic stimuli trigger apoptosis through a multimolecular complex critically involving APAF-1 and caspase 9 and ultimately leading to caspase 3 activation and apoptosis. In our study, the pU2-transfected cells showed significant increases in caspase activity.

We also used the MitoPT assay to further confirm apoptotic effects of pU2. The MitoPT assay follows the principle that the staining dye enters the mitochondria and aggregates, which show red fluorescence. In contrast, in apoptotic cells where the mitochondrial membrane is compromised, the dye diffuses into the cytoplasm and is visualized as diffuse green fluorescence. In flow cytometry analysis, apoptosis leads to a drop in the number of cells showing red fluorescence when compared to healthy cells. We observed a drop in red fluorescent cells as well as a concomitant increase in small particulate matter, suggesting an increase in cellular debris in pU2-treated cells. The ratio of apoptotic and non-apoptotic cells clearly shows a 9-fold increase in the pU2-transfected cells from both cell lines indicating the efficacy of targeting the uPA/uPAR system in cancer cells.

The results clearly indicate that the uPAR/uPA system not only plays a significant role in the invasion of cancer cells, but also has a regulatory role in cell survival and proliferation. RNAi-induced simultaneous downregulation of these proteins has been shown to inhibit the activation of the pro-survival kinases phospho-ERK and phospho-p38, and possibly inhibit the release of angiogenic factors, leading to reduced angiogenesis. Significantly, the apoptotic cascade is triggered leading to cell death. The relative ease of handling and delivery of siRNA constructs in plasmid vectors when compared to adenoviral vectors and the specific downregulation of genes of interest presents an effective therapeutic tool against breast cancer.

\section{Acknowledgements}

We would like to thank Shellee Abraham for preparing the manuscript and Sushma Jasti and Diana Meister for reviewing the manuscript. This study was supported by N.I.H. grants CA75557, CA92393, CA95058, CA116708 and N.I.N.D.S. NS47699 and by Caterpillar Inc. and OSF St. Francis Inc., Peoria, IL (J.S.R.).

\section{References}

1. Symes K and Mercola M: Embryonic mesoderm cells spread in response to platelet-derived growth factor and signaling by phosphatidylinositol 3-kinase. Proc Natl Acad Sci USA 93: 9641-9644, 1996.

2. Blasi F: uPA, UPAR, PAI-1: key intersection of proteolytic, adhesive and chemotactic highways? Immunol Today 18: 415-417, 1997.

3. Leirisalo-Repo M: The present knowledge of the inflammatory process and the inflammatory mediators. Pharmacol Toxicol 75 (Suppl 2): 1-3, 1994.

4. Camussi G, Montrucchio G, Lupia E, Soldi R, Comoglio PM and Bussolino F: Angiogenesis induced in vivo by hepatocyte growth factor is mediated by platelet-activating factor synthesis from macrophages. J Immunol 158: 1302-1309, 1997.

5. Kim HJ, Ingbar DH and Henke CA: Integrin mediation of type II cell adherence to provisional matrix proteins. Am J Physiol 271: L277-L286, 1996.

6. Aznavoorian S, Murphy AN, Stetler-Stevenson WG and Liotta LA: Molecular aspects of tumor cell invasion and metastasis. Cancer 71: 1368-1383, 1993.

7. Sato H, Takino T, Okada Y, Cao J, Shinagawa A, Yamamoto E and Seiki M: A matrix metalloproteinase expressed on the surface of invasive tumour cells. Nature 370: 61-65, 1994.

8. Stetler-Stevenson WG, Aznavoorian S and Liotta LA: Tumor cell interactions with the extracellular matrix during invasion and metastasis. Annu Rev Cell Biol 9: 541-573, 1993.

9. Vassalli JD and Pepper MS: Tumour biology. Membrane proteases in focus. Nature 370: 14-15, 1994.

10. Mazar AP: The urokinase plasminogen activator receptor (UPAR) as a target for the diagnosis and therapy of cancer. Anticancer Drugs 12: 387-400, 2001.

11. Ossowski L and Aguirre-Ghiso JA: Urokinase receptor and integrin partnership: coordination of signaling for cell adhesion, migration and growth. Curr Opin Cell Biol 12: 613-620, 2000.

12. Preissner KT, Nawroth PP and Kanse SM: Vascular protease receptors: integrating haemostasis and endothelial cell functions. J Pathol 190: 360-372, 2000.

13. Dano K, Romer J, Nielsen BS, Bjorn S , Pyke C, Rygaard J and Lund LR: Cancer invasion and tissue remodeling - cooperation of protease systems and cell types. APMIS 107: 120-127, 1999.

14. Mustjoki S, Alitalo R, Stephens RW and Vaheri A: Plasminogen activation in human leukemia and in normal hematopoietic cells. APMIS 107: 144-149, 1999.

15. Yu W, Kim J and Ossowski L: Reduction in surface urokinase receptor forces malignant cells into a protracted state of dormancy. J Cell Biol 137: 767-777, 1997.

16. Moller LB: Structure and function of the urokinase receptor. Blood Coagul Fibrinolysis 4: 293-303, 1993.

17. Stahl A and Mueller BM: Melanoma cell migration on vitronectin: regulation by components of the plasminogen activation system. Int J Cancer 71: 116-122, 1997.

18. Wei Y, Lukashev M, Simon DI, Bodary SC, Rosenberg S, Doyle MV and Chapman HA: Regulation of integrin function by the urokinase receptor. Science 273: 1551-1555, 1996.

19. Burgering BM and Bos JL: Regulation of Ras-mediated signalling: more than one way to skin a cat. Trends Biochem Sci 20: 18-22, 1995 .

20. Elbashir SM, Harborth J, Lendeckel W, Yalcin A, Weber K and Tuschl T: Duplexes of 21-nucleotide RNAs mediate RNA interference in cultured mammalian cells. Nature 411: 494-498, 2001.

21. Zamore PD: RNA interference: listening to the sound of silence. Nat Struct Biol 8: 746-750, 2001.

22. Lakka SS, Gondi CS, Yanamandra N, Olivero WC, Dinh DH, Gujrati M and Rao JS: Inhibition of cathepsin B and MMP-9 gene expression in glioblastoma cell line via RNA interference reduces tumor cell invasion, tumor growth and angiogenesis. Oncogene 23: 4681-4689, 2004. 
23. Ma Z, Webb DJ, Jo M and Gonias SL: Endogenously produced urokinase-type plasminogen activator is a major determinant of the basal level of activated ERK/MAP kinase and prevents apoptosis in MDA-MB-231 breast cancer cells. J Cell Sci 114: 3387-3396, 2001.

24. Mohan PM, Lakka SS, Mohanam S, Kin Y, Sawaya R, Kyritsis AP, Nicolson GL and Rao JS: Downregulation of the urokinase-type plasminogen activator receptor through inhibition of translation by antisense oligonucleotide suppresses invasion of human glioblastoma cells. Clin Exp Metastasis 17: 617-621, 1999.

25. Mohanam S, Sawaya R, McCutcheon I, Ali-Osman F, Boyd D and Rao JS: Modulation of in vitro invasion of human glioblastoma cells by urokinase-type plasminogen activator receptor antibody. Cancer Res 53: 4143-4147, 1993.

26. Mohan PM, Chintala SK, Mohanam S, Gladson CL, Kim ES, Gokaslan ZL, Lakka SS, Roth JA, Fang B, Sawaya R, Kyritsis AP and Rao JS: Adenovirus-mediated delivery of antisense gene to urokinase-type plasminogen activator receptor suppresses glioma invasion and tumor growth. Cancer Res 59: 3369-3373, 1999.

27. Guo Y, Higazi AA, Arakelian A, Sachais BS, Cines D, Goldfarb RH, Jones TR, Kwaan H, Mazar AP and Rabbani SA: A peptide derived from the nonreceptor binding region of urokinase plasminogen activator (UPA) inhibits tumor progression and angiogenesis and induces tumor cell death in vivo. FASEB J 14: 1400-1410, 2000.

28. Huang S, New L, Pan Z, Han J and Nemerow GR: Urokinase plasminogen activator/urokinase-specific surface receptor expression and matrix invasion by breast cancer cells requires constitutive p38alpha mitogen-activated protein kinase activity. J Biol Chem 275: 12266-12272, 2000.

29. Bertrand JR, Pottier M, Vekris A, Opolon P, Maksimenko A and Malvy C: Comparison of antisense oligonucleotides and siRNAs in cell culture and in vivo. Biochem Biophys Res Commun 296: 1000-1004, 2002.

30. Stark JJ, Dillman RO, Schulof R, Wiemann MC, Barth NM, Honeycutt PJ and Soori G: Interferon-alpha and chemohormonal therapy for patients with advanced melanoma: final results of a phase I-II study of the Cancer Biotherapy Research Group and the Mid-Atlantic Oncology Program. Cancer 82: 1677-1681, 1998.

31. Li H, Lu H, Griscelli F, Opolon P, Sun LQ, Ragot T, Legrand Y, Belin D, Soria J, Soria C, Perricaudet M and Yeh P: Adenovirusmediated delivery of a uPA/uPAR antagonist suppresses angiogenesis-dependent tumor growth and dissemination in mice. Gene Ther 5: 1105-1113, 1998.

32. Min HY, Doyle LV, Vitt CR, Zandonella CL, Shuman MA, Stratton-Thomas JR and Rosenberg S: Urokinase receptor antagonists inhibit angiogenesis and primary tumor growth in syngeneic mice. Cancer Res 56: 2428-2433, 1996.

33. Busso N, Masur SK, Lazega D, Waxman S and Ossowski L: Induction of cell migration by pro-urokinase binding to its receptor: possible mechanism for signal transduction in human epithelial cells. J Cell Biol 126: 259-270, 1994.

34. Fazioli F, Resnati M, Sidenius N, Higashimoto Y, Appella E and Blasi F: A urokinase-sensitive region of the human urokinase receptor is responsible for its chemotactic activity. EMBO J 16: 7279-7286, 1997.

35. Fibbi G, Ziche M, Morbidelli L, Magnelli L and Del Rosso M: Interaction of urokinase with specific receptors stimulates mobilization of bovine adrenal capillary endothelial cells. Exp Cell Res 179: 385-395, 1988.

36. Nguyen DH, Hussaini IM and Gonias SL: Binding of urokinasetype plasminogen activator to its receptor in MCF-7 cells activates extracellular signal-regulated kinase 1 and 2 which is required for increased cellular motility. J Biol Chem 273: 8502-8507, 1998.
37. Resnati M, Guttinger M, Valcamonica S, Sidenius N, Blasi F and Fazioli F: Proteolytic cleavage of the urokinase receptor substitutes for the agonist-induced chemotactic effect. EMBO J 15: $1572-1582,1996$.

38. Sato Y and Rifkin DB: Autocrine activities of basic fibroblast growth factor: regulation of endothelial cell movement, plasminogen activator synthesis, and DNA synthesis. J Cell Biol 107: 1199-1205, 1988.

39. Sato $Y$, Tsuboi R, Lyons R, Moses $H$ and Rifkin DB: Characterization of the activation of latent TGF-beta by cocultures of endothelial cells and pericytes or smooth muscle cells: a self-regulating system. J Cell Biol 111: 757-763, 1990.

40. Sidenius N and Blasi F: The urokinase plasminogen activator system in cancer: recent advances and implication for prognosis and therapy. Cancer Metastasis Rev 22: 205-222, 2003.

41. Chiaradonna F, Fontana L, Iavarone C, Carriero MV, Scholz G, Barone MV and Stoppelli MP: Urokinase receptor-dependent and -independent p56/59(hck) activation state is a molecular switch between myelomonocytic cell motility and adherence. EMBO J 18: 3013-3023, 1999.

42. Fazioli $F$ and Blasi F: Urokinase-type plasminogen activator and its receptor: new targets for anti-metastatic therapy? Trends Pharmacol Sci 15: 25-29, 1994.

43. Aguirre Ghiso JA, Kovalski K and Ossowski L: Tumor dormancy induced by downregulation of urokinase receptor in human carcinoma involves integrin and MAPK signaling. J Cell Biol 147: 89-104, 1999.

44. Liu D, Aguirre Ghiso JA, Estrada Y and Ossowski L: EGFR is a transducer of the urokinase receptor initiated signal that is required for in vivo growth of a human carcinoma. Cancer Cell 1: 445-457, 2002.

45. Tang H, Kerins, DM Hao, Q, Inagami T and Vaughan DE: The urokinase-type plasminogen activator receptor mediates tyrosine phosphorylation of focal adhesion proteins and activation of mitogen-activated protein kinase in cultured endothelial cells. J Biol Chem 273: 18268-18272, 1998

46. Rabbani SA, Gladu J, Mazar AP, Henkin J and Goltzman D: Induction in human osteoblastic cells ( $\mathrm{SaOS} 2)$ of the early response genes fos, jun, and myc by the amino terminal fragment (ATF) of urokinase. J Cell Physiol 172: 137-145, 1997.

47. Kjoller L and Hall A: Rac mediates cytoskeletal rearrangements and increased cell motility induced by urokinase-type plasminogen activator receptor binding to vitronectin. J Cell Biol 152: 1145-1157, 2001

48. Bonni A, Brunet A, West AE, Datta SR, Takasu MA and Greenberg ME: Cell survival promoted by the Ras-MAPK signaling pathway by transcription-dependent and -independent mechanisms. Science 286: 1358-1362, 1999.

49. Guillonneau X, Bryckaert M, Launay-Longo C, Courtois Y and Mascarelli F: Endogenous FGF1-induced activation and synthesis of extracellular signal-regulated kinase 2 reduce cell apoptosis in retinal-pigmented epithelial cells. J Biol Chem 273: 22367-22373, 1998 .

50. Xia Z, Dickens M, Raingeaud J, Davis RJ and Greenberg ME: Opposing effects of ERK and JNK-p38 MAP kinases on apoptosis. Science 270: 1326-1331, 1995.

51. Zou H, Henzel WJ, Liu X, Lutschg A and Wang X: Apaf-1, a human protein homologous to $C$. elegans CED-4, participates in cytochrome c-dependent activation of caspase-3. Cell 90: 405-413, 1997.

52. Cecconi F, Alvarez-Bolado G, Meyer BI, Roth KA and Gruss P: Apaf1 (CED-4 homolog) regulates programmed cell death in mammalian development. Cell 94: 727-737, 1998. 